\title{
Freiwillige Klimakompensation als grünes Produkt?
}

\author{
Der Markt für freiwillige Zahlungen zur Kompen- \\ sation von Treibhausgasemissionen wächst \\ weltweit. Untersuchungen zeigen, dass frei- \\ willige Kompensationen einen guten Beitrag zum \\ Klimaschutz leisten können. Ein staatliches \\ Gütesiegel könnte die Markttransparenz und \\ die Akzeptanz verbessern. \\ Von Stephan Wolters und Romy Becker
}

K limaschutz ist ein öffentliches Gut. Der wirtschaftliche Anreiz für eigene Anstrengungen und der direkte persönliche Nutzen sind gering. Gleichzeitig profitiert man von den Maßnahmen anderer, das sogenannte Freeriding (Goldemberg et al. 1996). Zahlreiche empirische Studien belegen aber, dass dennoch freiwillige Klimaschutzaktivitäten von Individuen durchgeführt werden. Eine solche indirekte Klimaschutzaktivität ist die finanzielle Kompensation von $\mathrm{CO}_{2}$-Emissionen, die beim eigenen Konsum von Gütern und Dienstleistungen entstehen, dem sogenannten Offsetting.

Das Prinzip der Kompensation beruht auf dem Gedanken, dass es für das Klima nicht entscheidend ist, an welcher Stelle Treibhausgase ausgestoßen oder vermieden werden. Daher lassen sich an einer Stelle verursachte Emissionen an anderer Stelle einsparen. Das globale Emissionsniveau wird bei der Kompensation allerdings nicht reduziert, da netto keine Emissionen vermieden werden. Zum freiwilligen Ausgleich wird zunächst die Höhe der klimawirksamen Emissionen einer bestimmten Aktivität berechnet. Der Ausgleich erfolgt dann über Emissionsgutschriften in Form von Zertifikaten, mit denen dieselbe Emissionsmenge in Klimaschutzprojekten ausgeglichen wird, die es ohne den Mechanismus der Kompensation nicht gegeben hätte.

\section{Wie entwickelt sich der deutsche Markt?}

Immer mehr Menschen und Unternehmen wollen, dass für von ihnen verursachte klimaschädliche Emissionen an anderer Stelle entsprechende Treibhausgase vermieden werden. Am häufigsten tun sie dies mit Kompensationen für Flugreisen. Unternehmen, die etwa $80 \%$ der Nachfrage stellen, setzen die meisten Zertifikate zur Kompensation ihres unternehmerischen oder eines produktspezifischen Fußabdrucks ein. So stieg das Volumen sogenannter stillgelegter Zertifikate, also solcher, die dem Handel dauerhaft entzogen werden, auf dem deutschen Markt von 2012 zu 2013 um etwa 33\% auf 4,4 Millionen Tonnen Kohlenstoffdioxid-Äquivalente $\left(\mathrm{MtCO}_{2 \mathrm{e}}\right.$ ) (Wolters et al. 2015a). Damit setzt sich der Aufwärtstrend der letzten Jahre fort (Kind et al. 2010). Der deutsche Markt zeigt sich damit robust in einem schwierigen internationalen Marktumfeld. Die Umsätze gingen auf geschätzte 379 Millionen US-Dollar zurück, was sowohl geringeren Volumina als auch geringeren Durchschnittspreisen zuzuschreiben ist (Ecosystem Marketplace 2014).

Der deutsche Markt hat sich dynamisch entwickelt und ausdifferenziert. Das Preisniveau schwankt erheblich. Abhängig vom genutzten Qualitätsstandard reicht die Spanne von 40 Cent bis zu 50 Euro pro Tonne $\mathrm{CO}_{2}$. Der Preis der Zertifikate korreliert unter anderem stark mit dem Verkaufsvolumen, ist aber auch als Indiz für einen bisher noch intransparenten Markt zu werten. Die niedrigsten Preise werden für Certified Emission Reductions (CERs) und Voluntary Carbon Standard (VCS), die höchsten für deutsche Moorschutz-Zertifikate und Gold Standard CERs erzielt. Auffällig ist, dass die Preisprämie für die Kombination von VCS mit Zusatzstandards für strengere Nachhaltigkeitskriterien relativ gering ausfällt. Der VCS, auch in Kombination mit Zusatzstandards, deckt dabei den größten Marktanteil mit rund 57\% in 2013 ab (Wolters et al. 2015a).

\section{Was sagen die Verbraucher?}

Insgesamt haben etwa $10 \%$ der deutschen Bevölkerung schon einmal Kompensationsmaßnahmen durchgeführt. Nur $25 \%$ können sich dies für die Zukunft nicht vorstellen. Es

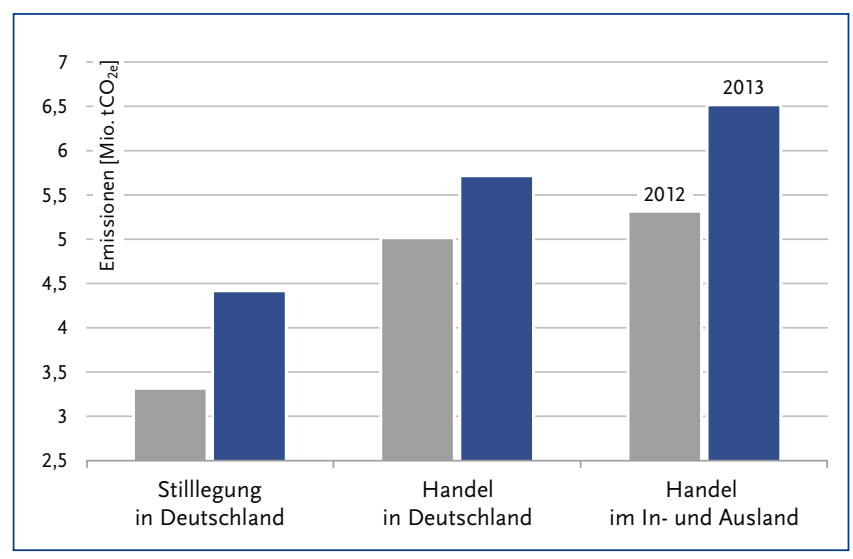

Abbildung 1: Emissionszertifikate auf dem freiwilligen Markt von 2012-2013 Quelle: Wolters et al. 2015a 
besteht also ein erhebliches Potenzial (Schwirplies/Ziegler 2014).

Als wichtigstes Kriterium für die Kaufentscheidung nannten $36 \%$ der gut 200 Teilnehmer(innen) einer nicht repräsentativen Umfrage die Klima- und Umweltwirkung der durch die Zertifikate unterstützten Projekte. Dieser Aspekt wurde auch insgesamt überdurchschnittlich häufig als zweite und dritte Priorität genannt. Etwa 30\% der Unternehmen, aber nur rund 3\% anderer Nachfragergruppen nennen den Preis als wichtigstes Kriterium. Die Förderung sozialer und Nachhaltigkeitsaspekte im Herkunftsland spielt ebenfalls eine wichtige Rolle. $17 \%$ der Nachfrager(innen) führen sie als wichtigstes Kaufargument auf. Insbesondere als sekundäre oder tertiäre Entscheidungsgröße beeinflusst sie den Zertifikatskauf. Ihre wichtige Rolle spiegelt sich auch im Wunsch von fast $60 \%$ der Verbraucher wieder, Zertifikate von gemeinnützigen Anbietern zu beziehen. Gleichwohl liegt der tatsächliche Marktanteil solcher Anbieter am stillgelegten Volumen bei lediglich 15\% (Wolters et al. 2015a).

Als weiteres Entscheidungskriterium folgt die Qualität des verwendeten Standards, die für 15\% der Nachfrager ausschlaggebend ist. Verbreitete Standards mit hohen Anforderungen werden so gut beziehungsweise besser als CERs bewertet. In Deutschland hat insbesondere der Gold Standard eine hohe Reputation. Die Nutzbarkeit von CERs für den freiwilligen Markt ist allerdings noch nicht hinlänglich bekannt (Wolters et al. 2015a).

Interessant ist der Vergleich der Kaufkriterien der Nachfrager mit den Einschätzungen der Anbieter. Den Einschätzungen zufolge ist der Qualitätsstandard das wichtigste Kaufkriterium. Nachfrager nennen diesen Aspekt erst an vierter Stelle. Umwelt- und Klimawirkung, das Top-Kriterium für Nachfrager, nennen Anbieter hingegen erst nachrangig. Das muss jedoch kein Widerspruch sein, sondern zeigt vielmehr auf, dass Qualitätsstandards gewissermaßen der Zertifikats-Transmissionsmechanismus der Umwelt- und Klimawirkung sind. Aus Anbieter-Perspektive erweist sich der Qualitätsstandard daher als das entscheidende Kaufkriterium der Kund(inn)en (Wolters et al. 2015a).

Fast $80 \%$ der Projekte kommen aus dem Bereich der erneuerbaren Energien. Es folgen Forst- und Landwirtschaft mit $15 \%$. Allerdings sprechen sich nur $42 \%$ der Nachfrager für erneuerbare Energien als favorisierten Projekttyp aus und 35\% für Energieeffizienz. Das sind derzeit nur 5\% des Volumens. Die meisten Zertifikate stammen dabei aus Asien und Pazifik und damit aus einer Region, die bei den Nachfrager-Präferenzen hinter Afrika und dem Nahen Osten nur auf Platz drei rangiert. Fast 50\% der Nachfrager geben hingegen Deutschland als erste Präferenz für das Herkunfts- land der Zertifikate an. Diesem Anteil stehen aufgrund eines Unterangebots an Projekten aus Deutschland nur knapp $10 \%$ der tatsächlichen Stilllegungen gegenüber (Wolters et al. 2015a).

\section{Verdrängen Kompensationsmaßnahmen Klimaschutzaktivitäten?}

Freiwillige Treibhauskompensation kann als weiteres Klimaschutzinstrument maßgeblich zur effizienten Vermeidung von Emissionen beitragen, ohne dass dies gegenüber dem Verpflichtungsmarkt notwendiger Weise mit Qualitätseinbußen einhergehen muss.

Doch das Instrument der Kompensation ist auch umstritten, denn $\mathrm{CO}_{2}$-Offsetting könnte andere Klimaschutzaktivitäten verdrängen. Kompensation könnte Menschen dazu bewegen, sich um ihren eigenen ökologischen Fußabdruck nicht mehr zu sorgen. In dem Zusammenhang stellen manche die Moralität der Kompensation infrage, da man sich von den eigenen Klimasünden gewissermaßen freikaufen könne. Dieser Effekt könnte sogar zu höheren $\mathrm{CO}_{2}$-Emissionen führen im Vergleich zu einer Welt ohne freiwillige Kompensation (Schwirplies/Ziegler 2014).

Einer Studie zufolge ergibt sich insgesamt aber ein positiver Zusammenhang zwischen der Bereitschaft für $\mathrm{CO}_{2}$-Offsetting und anderen direkten Klimaschutzaktivitäten, die insbesondere durch finanzielle Vorteile beeinflusst werden. Das bedeutet, dass $\mathrm{CO}_{2}$-Offsetting andere direkte Klimaschutzaktivitäten nicht verdrängt beziehungsweise klimaschädigende Maßnahmen nicht erhöht. Offsetting geht sogar mit mehr Klimaschutz einher. Eine Verdrängung findet also nicht statt. Insofern spricht aus Sicht des Klimaschutzes nichts gegen die Unterstützung solcher Maßnahmen als einen Baustein der Klimapolitik. $\mathrm{CO}_{2}$-Offsetting ist kein Ablasshandel, sondern führt tatsächlich zu mehr Klimaschutz. Haushalte sind, trotz möglicher hypothetischer Verzerrungen bei der Befragung, zunehmend 


\section{„Für den Erfolg von \\ freiwilligen Kompensationen \\ ist eine transparente \\ Kommunikation entscheidend."}

Der Skepsis der Nachfragenden könnte durch Formen staatlicher Zertifizierung entgegengewirkt werden. Um die Marktintransparenz zu adressieren, könnte ein staatliches Label, als eine Art Gütesiegel ähnlich dem Blauen Engel für Umweltschutzstandards, einheitlich hohe Qualitätsanforderungen sicherstellen und damit den Kompensationsmarkt transparenter gestalten. Marktakteure bewerten ein mögliches Label als grundsätzlich wünschenswert. Allerdings kann das erhebliche Transaktionskosten mit sich bringen. Auf einem stark international ausgerichteten Markt könnten daher erhebliche Synergieeffekte erzielt werden, wenn ein solches Vorgehen über Ländergrenzen hinaus abgestimmt wird.

\section{Wie lässt sich freiwillige Kompensation kommunizieren?}

mit auseinanderzusetzen (Lange et al. 2014).

Eine Online-Befragung von 566 Privatpersonen mit einem Umweltschutzhintergrund ergab, dass je besser das allgemeine Umweltverhalten und je besser das Wissen zum Thema Kompensation sind, desto mehr wird kompensiert. Die meisten Umweltschützer(innen) haben eher eine differenzierte Meinung zum Thema Kompensation. Eine klar ablehnende Meinung gibt es nur bei einer Minderheit (Locher 2014).

Personen, die klimaschädliche Emissionen ihres Konsumverhaltens kompensieren, pflegen also tendenziell auch anderweitig einen nachhaltigeren Lebensstil. Das bedeutet, dass Kompensationszahlungen keineswegs als Freikauf oder Freibrief, sondern als eine zusätzliche Möglichkeit für klimaschützendes Handeln verstanden werden. Nachhaltige Lebensstile und freiwillige Kompensation passen also gut zusammen.

Die Broschüre „Klimaneutral leben“ des Umweltbundesamtes zeigt zudem auf, wie sich diese Erkenntnis strategisch für die Umweltkommunikation nutzen lässt (Umweltbundesamt 2014). Um das Instrument der freiwilligen Kompensation von $\mathrm{CO}_{2}$-Emissionen weiter $\mathrm{zu}$ fördern, sollte eine positive Form der Kommunikation gewählt und der Mechanismus in der Öffentlichkeit nicht übermäßig problematisiert werden. Internes Lobbying beispielsweise bei der öffentlichen Beschaffung könnte als weiteres Signal für Verbraucher(innen) dienen, das die Vorteile von Kompensationen in den Vordergrund rückt (Wolters et al. 2015b).

Dazu passt auch, dass nur relativ wenige Nachfrager Umfrageergebnissen zufolge Kompensationsmaßnahmen als Freikauf oder Ablasshandel betrachten. Nur für deutlich unter 20\% ist dies der entscheidende Grund, keine Kompensation durchzuführen (Wolters et al. 2015a).

\section{Brauchen wir ein staatliches Label?}

Eine viel größere Rolle spielen der gleichen Umfrage zufolge finanzielle Erwägungen und Marktintransparenz. Insgesamt bestehen deutliche Informationsdefizite. Dies unterstreicht die Wichtigkeit und Notwendigkeit von Aufklärungsund Informationskampagnen (Schwirplies/Ziegler 2014).
Ein nachhaltiger Lebensstil sowie Fragen des Umweltschutzes etablieren sich zunehmend in der Gesellschaft. Laut der Umweltbewusstseinsstudie von 2014 sind über die Hälfte der Befragten der Meinung, dass Umweltschutz eine grundlegende Bedingung ist, um zukünftige Herausforderungen zu meistern und den Wohlstand zu sichern (Bundesministerium für Umwelt, Naturschutz, Bau und Reaktorsicherheit 2015). Innovative Konzepte, die geeignet sind, einen Beitrag zu Umweltund Klimaschutz zu leisten, und gleichzeitig zu einer hohen, aber vom Ressourcenverbrauch unabhängigen Lebensqualität führen, stoßen auf großes Interesse bei den Befragten (ebd.). Ebenso äußern über $80 \%$ der Befragten das Bedürfnis, mit sich und der Umwelt im Reinen zu leben. Darüber hinaus wird die globale Dimension der eigenen Lebensweise wahrgenommen. Neun von zehn Befragten geben an, dass die Lebensweise in Deutschland für Umweltprobleme in anderen Ländern verantwortlich ist (ebd.).

Demzufolge müsste die Kommunikation für die freiwillige Kompensation gegenüber den Verbraucher(inne)n auf fruchtbaren Boden stoßen. Denn es besteht noch Potenzial in der Wahrnehmung um die Möglichkeiten der freiwilligen Kompensation und in der Motivation, diese Möglichkeiten in seinem alltäglichen Handeln umzusetzen. Denn, wie bereits erwähnt, schließt die freiwillige Kompensation den Klimaschutz nicht aus. Doch wie bei jeder anderen Kommunikationsstrategie für nachhaltige Handlungsempfehlungen stellen sich auch hier die folgenden Fragen: Wer ist die Hauptzielgruppe? Wer sind Multiplikatoren? Was sind die zentralen Fragestellungen und

Die zielgerichtete Kommunikation über freiwillige Kompensation könnte verstärkt bei Verbraucher(inne)n ansetzen, die bereits umweltschützende Aspekte in ihrem Lebensstil berücksichtigen, um vertiefend und aktivierend über deren Vorteile $\mathrm{zu}$ informieren. Denn je besser das allgemeine Umweltverhalten und je besser das Wissen zum Thema Kompensation ist, desto mehr wird kompensiert (Locher 2014). Zentral wäre dabei unter anderem die Berücksichtigung der folgenden Nutzenargumente in den Kernbotschaften (ebd.): Nutzenargumente? Und wie sollen diese transportiert werden? 
- Mit sich und der Umwelt in Einklang leben. Durch die freiwillige Kompensation können statt Kilogramm Tonnen an Treibhausgasen vermieden werden.

I Die Zukunft meistern. Kompensationsprojekte befördern im Normalfall investive Maßnahmen, die langfristig in ihrer Wirkung sind und Infrastrukturen kohlenstoffleichter gestalten.

I Einen Beitrag leisten. Freiwillige Kompensationszahlungen fördern einerseits Effizienzmaßnahmen. Andererseits bedeuten sie für den Zahlenden einen Verzicht, indem sie das Geld verschenken, dass sie an anderer Stelle für den Konsum von anderen Gütern einsetzen würden. Zugespitzt ausgedrückt sind sie damit letztlich auch eine Maßnahme der Suffizienz.

Für eine transparente Kommunikation gegenüber der Zielgruppe wäre auch die Klärung der folgenden Aspekte notwendig (Bilharz/Becker 2015):

IVerbraucher(innen) bilden und schützen. Wie lässt sich Verbrauchertäuschung entgegenwirken? Was bietet Orientierung bei der Entscheidung zur freiwilligen Kompensation? Wie können Kompensationsdienstleister mit ihrem Knowhow als Bildungsakteure gegenüber den Verbraucher(inne)n fungieren?

I Kampf den schwarzen Schafen. Wie kann Greenwashing offengelegt werden? Wie kann verhindert werden, dass nicht treibhausgasinduzierte Umweltschäden durch $\mathrm{CO}_{2}$-Kompensation kaschiert werden?

I Qualität auf dem Prüfstand. Welche Standards braucht es? Ist ein Label sinnvoll?

I Stärkung vorbildlicher Kompensationsdienstleister. Wer sind die Good-Practice-Beispiele bei den Marktplayern? Wie können diese gestärkt werden?

Zudem stellt sich die Frage nach der strategischen Kommunikation von freiwilliger Kompensation. Bisher ist diese oftmals an Produkte wie Flugreisen, Paketversand oder Bahnreisen sowie an Dienstleistungen und Veranstaltungen gebunden. Der Klimaschutz wird somit über die Marketingkanäle der Produkte publik gemacht. Doch ist die Bindung an ein konkretes Produkt für die freiwillige Kompensation zwingend notwendig? Die produktunabhängige Kompensation des persönlichen oder durchschnittlichen Jahresausstoßes von Treibhausgasen könnte ebenso kommuniziert werden (Bilharz/Becker 2015). Denkbar wäre dies hier in Form von Sensibilisierungskampagnen in der Zeit rund um die jährlichen Klimagipfel (ebd.). Die Kampagnen würden positiv und aktivierend zu Möglichkeiten der freien Kompensation informieren, die stark im Alltag der Zielgruppe ansetzen.

\section{Kompensation unterstützt nachhaltige Lebensstile}

Freiwillige Kompensation macht andere Klimaschutzmaßnahmen nicht überflüssig, sondern kann diese ergänzen. Sie kann zudem, wie andere freiwillige Maßnahmen auch, grund- legende politische Änderungen und Vorgaben auf nationaler und internationaler Ebene nicht ersetzen, sondern sollte diesen möglichst den Boden bereiten.

Freiwillige Kompensation ist keineswegs als Strohfeuer anzusehen. Vielmehr weisen empirische Ergebnisse darauf hin, dass Kompensation nachhaltig klimaschützende Effekte erzielt. Freiwillige Kompensation sollte daher als effektive Handlungsoption für Verbraucher(innen) weitere Verbreitung finden. Dazu ist vor allem transparente und kompetente Kommunikation erforderlich.

\section{Literatur}

Bilharz, M./Becker, R. (2015): Freiwillige Kompensation: Ein heißes Eisen für die Umweltkommunikation? In: Wolters, S./Nett, K./Schindler, H. (2015b): Freiwillige Kompensationszahlungen und nachhaltige Lebensstile: Passt das zusammen? Dokumentation der UBA-Tagung am 10. November 2014 in Berlin. Berlin.

Bundesministerium für Umwelt, Naturschutz, Bau und Reaktorsicherheit (2015): Umweltbewusstsein 2014: Ergebnisse einer repräsentativen Bevölkerungsumfrage. Berlin.

Ecosystem Marketplace (2014): Sharing the Stage: State of the Voluntary Carbon Markets 2014. Washington, D.C.

Goldemberg, J. et al. (1996): Introduction: scope of the assessment. In: Bruce, J. P. et al.: Climate Change 1995: Economic and Social Dimensions of Climate Change. Contribution of Working Group III to the Second Assessment Report of the Intergovernmental Panel on Climate Change. Cambridge.

Kind, C. et al. (2010): Analyse des deutschen Marktes zur freiwilligen Kompensation von Treibhausgasemissionen. Studie im Auftrag der Deutschen Emissionshandelsstelle im Umweltbundesamt. Climate Change Nr. 10/2010.

Lange, A./Schwirplies, C./Ziegler, A. (2014): On the interrelation between carbon offsetting and other voluntary climate protection activities: Theory and empirical evidence. MAGKS Discussion Paper 47.

Locher, M. (2014): Umweltschützer und freiwillige Kompensation: Ergebnisse einer UBA-Studie. Präsentation zur Tagung Freiwillige Kompensationszahlungen und nachhaltige Lebensstile: Passt das zusammen?

Schwirplies, C./Ziegler, A. (2014): Offset emissions or pay a premium for avoiding them? A cross-country analysis of motives for voluntary climate protection activities. Unveröffentlichtes Manuskript.

Umweltbundesamt (2014): Klimaneutral leben. Verbraucher starten durch beim Klimaschutz. Dessau-Roßlau.

Wolters, S./Nett, K./Tänzler, D./Wilkening, K./Götz, M./Krebs, J.-M./Vogel, D. (2015a): Aktualisierte Analyse des deutschen Marktes zur freiwilligen Kompensation von Treibhausgasemissionen. Berlin.

Wolters, S./Nett, K./Schindler, H. (2015b): Freiwillige Kompensationszahlungen und nachhaltige Lebensstile: Passt das zusammen? Dokumentation der UBA-Tagung am 10. November 2014 in Berlin. Berlin.

\section{AUTOREN + KONTAKT}

Stephan Wolters arbeitet als Projektmanager in der Klima- und Energiepolitik bei adelphi.

Tel.: +49 30 8900068-29, E-Mail: wolters@adelphi.de

Romy Becker verantwortet als Senior Projektmanagerin bei adelphi Dialog- und Beratungsvorhaben in den Bereichen Politik und Wirtschaft sowie Unternehmen und Finanzen. Tel.: +49 30 8900068-27, E-Mail: becker@adelphi.de

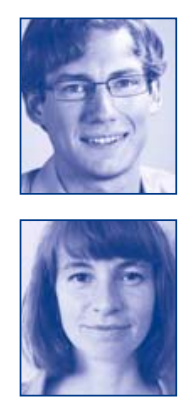

